\title{
Les cantiques daniéliques (Dn 3,26-90) vieux latins et leurs types de texte
}

Par

\section{Jean-Claude Haelewyck ${ }^{*}$}

Université catholique de Louvain, Louvain-la-Neuve

L

e livre de Daniel a livré deux grands cantiques bibliques : le cantique d'Azarias (Dn 3,26-45) et le cantique des Trois Jeunes Gens (Dn 3,57-88[90]). Un troisième, plus (Dn $3,52-56)$, vient s'inserer entre ces deux ensembles . le cantique de la transcendance divine (Dn 3,52-56) ${ }^{1}$, rattaché tantôt avec le précédent tantôt avec le suivant. Les deux grands cantiques font partie des suppléments grecs transmis à la fois par la Septante et par Théodotion². À ces pièces vient s'ajouter la prière de Daniel (Dn 9, 4-19) qui, elle, est commune à toutes les formes textuelles (texte hébreu massorétique et textes grecs). Elle apparaît parmi les dixhuit cantiques annexés au Psautier du Sinaï (VL 460), mais P. Bogaert a montré qu'il s'agit là d'une refonte liturgique du texte vulgate ${ }^{3}$, et à ce titre le passage n'entre pas dans le cadre de cette recherche.

* Je remercie Claude Obsomer et Daniel Malnati pour leur relecture attentive.

${ }^{1}$ Intitulé ainsi par $\mathrm{Ph}$. Bernard, voir infra.

${ }^{2}$ La refonte grecque du livre de Daniel, qui nous est parvenue sous le nom de Théodotion, n'a probablement pas grand-chose à voir avec le réviseur hexaplaire du même nom. Voir, parmi l'abondante littérature sur le sujet, l'étude fondamentale de SCHMITT 1966. Pour l'édition du grec, voir ZIEGLER, MUNNICH, FrAENKEL 1999.

${ }^{3}$ Bogaert 2004. 
L'objectif de la recherche qui suit est de tenter d'identifier les types de texte vieux latins du livre de Daniel tels qu'ils sont habituellement proposés dans les éditions de la Vetus Latina de Beuron. L'article se veut une recherche préparatoire à l'édition qui m'a été confiée. Les textes vieux latins sont transmis par deux voies principales : en tradition directe par des manuscrits (liturgiques ou non) et en tradition indirecte par les citations patristiques. D'un livre à l'autre, la proportion entre manuscrits et citations varie considérablement, comme me l'a montré mon expérience d'éditeur en ce domaine. Dans les cas d'Esther et de Judith, les manuscrits sont relativement nombreux, à l'inverse des citations patristiques. C'est mutatis mutandis aussi le cas pour l'Évangile de Marc. Pour ces trois livres, les types de texte transmis par les manuscrits sont clairement identifiables. Et les citations ne viennent que confirmer les conclusions tirées de l'analyse des témoins directs. Le cas du livre de Daniel est plus complexe. Si on met à part les cantiques du chapitre 3 , seuls trois témoins manuscrits nous font connaître le texte vieux latin de Daniel. Ce sont les manuscrits VL 175 (Italie du Nord, 5e s.), 176 (Saint-Gall, premières décennies du $9^{\mathrm{e}} \mathrm{s}$.) et 177 (écriture inférieure du palimpseste de Würzburg, Italie, $5^{\mathrm{e}}$ s.). Ces trois témoins sont fragmentaires, mais ils transmettent de très larges proportions du livre. Pour certaines d'entre elles, ils se recouvrent. Les passages qu'ils ont en commun permettent d'affirmer que ces témoins sont apparentés du point de vue textuel. Les citations patristiques sont en revanche plus nombreuses, en particulier pour la vision des quatre bêtes en Dn 7 et pour la prophétie des soixante-dix semaines au chapitre $9^{4}$. Et elles vont de Cyprien (milieu du $3^{\mathrm{e}} \mathrm{s}$.) jusqu'au $5^{\mathrm{e}} \mathrm{s}$., voire au-delà pour certaines d'entre elles. Grâce à ces citations, il est possible de situer dans le temps et dans l'espace le texte transmis par les trois manuscrits fragmentaires : il s'agit d'un texte européen du milieu du $4^{\mathrm{e}} \mathrm{s}$. Les témoins manuscrits des cantiques du chapitre 3 sont, eux, très nombreux, en particulier pour le cantique des Trois Jeunes Gens. Et dans le cas du cantique d'Azarias, ils augmentent considérablement notre connaissance des types de texte vieux latins du livre de Daniel. C'est ce que l'article qui suit voudrait montrer. Mais il faut d'abord commencer par présenter brièvement ce que l'on sait des cantiques de Daniel 3.

Dans l'Église latine, les premières mentions des cantiques daniéliques ${ }^{5}$ apparaissent à la fin du $4^{\mathrm{e}}$ et au début du $5^{\mathrm{e}} \mathrm{s}$. Dans les tout premiers témoignages, le lien avec la liturgie n'est cependant pas explicite. Ainsi Ambroise, dans son commentaire sur Luc composé vers 390, fait allusion (à propos de Lc 7,32 « nous avons chanté pour vous, mais vous n'avez pas dansé ») à une série de cantiques : le cantique de Moïse (Ex 15,1-20), le cantique de la Vigne (Is 5,1-7[9]), celui des Trois Jeunes Gens ${ }^{6}$ et celui d'Habacuc (Hab 3,1-19). Dans son Explanatio XII psalmorum (AM Ps, sur Ps 1,4-6, écrite entre 387 et 397$)^{7}$, il énumère les cantiques sui-

\footnotetext{
${ }^{4}$ Les citations patristiques peuvent être consultées sur le site http://www.brepolis.net/.

${ }^{5}$ L'étude fondamentale sur les cantiques bibliques, mais dépassée sur plus d'un point, reste celle de SCHNEIDER 1938, à compléter par ses trois articles : SCHNEIDER 1949a, 1949b et 1949c. On consultera aussi MEARNS 1914; DOLD 1947, et plus récement HARL 2014. Sur l'utilisation des cantiques dans l'homilétique en Afrique du Nord, voir BOGAERT 2010. Pour la tradition manuscrite, voir GRYSON 1999 et 2004, et surtout GRYSON 19931997, en particulier les p. 807-890 qui présentent et analysent les témoins manuscrits (liturgiques ou autres) des cantiques d'Isaïe dont certains sont transmis en même temps que ceux de Daniel.

${ }^{6}$ La phrase cantaverunt Hebraei cum vestigia eorum tactu flammae rorantis umescerent et intus extraque ardentibus omnibus solos tamen ignis innoxius lamberet nec adureret (AM Lc $6[177,66])$ semble indiquer qu'il ne s'agit que du Cantique des Trois Jeunes Gens (allusion à la rosée au v. 50), à l'exclusion de celui d'Azarias (Dn 3,26-45). Mais on peut hésiter, étant donné les liens étroits entre les deux pièces dans certains témoins, voir cidessous ce qui est dit de la liste de Nicétas.

${ }^{7}$ Les abréviations des œuvres patristiques latines sont reprises au répertoire de R. Gryson, qui indique aussi les éditions de référence.
} 
vants : le cantique de Moïse et de Myriam (Ex 15,1-20 + 15,21), le second de Moïse (Dt 32,143), celui de Déborah (Jg 5,1-31), le cantique de la Vigne, un cantique de Daniel et un cantique d'Habacuc ${ }^{8}$. Pélage, dans son commentaire des épîtres de Paul (entre 406 et 410), illustre les cantiques dont parle l'apôtre en Col 3,16 (« chantez à Dieu dans vos coeurs votre reconnaissance par des psaumes, des hymnes, des cantiques inspirés par l'Esprit») par les exemples des psaumes de David, du cantique des Trois Jeunes Gens et des deux cantiques de Moïse (Ex 15 et Dt 32) ${ }^{9}$. Augustin, vers 400, défend l'usage liturgique des divina cantica prophetarum, dont la récitation sobre suscite la réprobation des Donatistes partisans d'une célébration plus animée (AU ep 55,35) ${ }^{10}$. Dans ses œuvres Augustin cite le cantique d'Ex 15, celui de Jonas (Jon 2,3-9) et commente le cantique d'Azarias dans sa lettre 111. Au début du $5^{\mathrm{e}} \mathrm{s}$., on voit apparaître la première mention explicite d'une utilisation liturgique des cantiques. Nicétas ( $†$ 414), dans le De utilitate hymnorum 9, cite les deux cantiques de Moïse, celui d'Anne (1 Rg 2,1-10), le cantique De nocte (Is 26,9-20[21]), ceux d'Habacuc et de Jonas, un cantique de Jérémie (qui pourrait être Lm 5,1-22 selon l'hypothèse traditionnelle, ou Lm 3,1-9 ${ }^{11}$ ), celui des Trois Jeunes Gens et le Magnificat attribué à Elisabeth (Lc 1,46-55). Ces cantiques sont présentés par Nicétas comme des pièces chantées par l'Église de Dieu à l'office des Laudes ${ }^{12}$. Selon M. Harl, le cantique d'Azarias est très vraisemblablement aussi inclus dans la liste de Nicétas ${ }^{13}$. En effet, dit-elle, en 3,25, contrairement au texte de Théodotion où Azarias est seul à prier, la LXX affirme que ses compagnons étaient associés à la louange : «debout, Azarias priait...et confessait au Seigneur avec ses compagnons au milieu du feu de la fournaise...et ils disaient $»$. De plus, dans la tradition manuscrite grecque, certains psautiers font précéder les vv. 24-90, traités comme un ensemble, du titre « hymne - ou prière - des trois enfants » (mss T et 55 dans la liste de Rahlfs). Il en va de même dans la tradition latine, ainsi dans le Psautier Berlin, Hamilton 552 (VL 408) ou encore dans le Psautier du Sinaï (VL 460) ; ce dernier ne lit que les vv. 26-45 + 52-56 (sans le cantique des Trois Jeunes Gens donc) mais ceux-ci sont intitulés canticum trium puerorum. Rufin, dans son Apologie contre Jérôme, en 401, reproche à Jérôme d'avoir dans sa traduction totalement enlevé de sa place (e loco suo penitus erasum est) le cantique des Trois Jeunes Gens chanté depuis longtemps par toutes les Églises (RUF ap H 2,33 + 2,35). Cette affirmation polémique laisse perplexe. En effet, dans tous les témoins directs du texte vulgate, ainsi que dans les diverses listes de capitula, les cantiques d'Azarias et des Trois Jeunes Gens, munis d'obèles, sont bien situés au chapitre 3. Rien n'indique que Jérôme aurait déplacé le cantique à un autre endroit. Faudrait-il supposer que, dans une première édition non conservée, ce supplément aurait été placé en fin de livre comme dans le cas d'Esther? Rien ne le prouve. Pour l'Afrique du Nord, nous avons le témoignage de Verecundus de Junca (†552). Le seul manuscrit qui ait transmis

\footnotetext{
${ }^{8}$ Cette liste de cantiques pourrait représenter, d'après SCHNEIDER 1938, p. 10, la plus ancienne série milanaise. On observera toutefois qu'en tradition directe les témoins milanais ont conservé un autre choix de cantiques : Dn 3,52ss (cantique de la transcendance, voir infra), Is 26, $1 \mathrm{Rg}$ 2, Hab 3, Jon 2, Lc 1,68-79 (Zacharie), Dt 32, Ex 15, Dn 3,57-88 et Lc 1,46-54 (Magnificat).

${ }^{9}$ SOUTER 1926, p. 468.

10 ita ut Donatistae nos reprehendant, quod sobrie psallimus in ecclesia divina cantica prophetarum, cum ipsi ebrietates suas ad canticum psalmorum humano ingenio compositorum quasi ad tubas exhortationis inflamment.

${ }^{11}$ HARL 2014, p. 263-269. Pour l'hypothèse traditionnelle, voir MEARNS 1914, p. 56 ; SCHNEIDER 1938, p. 8, 21, 144.

12 NIC ut 1. Voir aussi Ambroise, Expositio de Psalmo CXVIII, 19,32.

${ }^{13}$ HARL 2014, p. 259-262.
} 
le commentaire de Verecundus est défiguré par une lacune : le commentaire du cantique d'Ézéchias s'interrompt sur Is 38,13 et il est suivi du commentaire d'Hab 3,4. On a de bonnes raisons de supposer qu'il contenait également au moins le cantique d'Is $26^{14}$. L'ouvrage, qui a dû être composé après 534, devait commenter les cantiques suivants : Ex 15,1-19; Dt 32,132, Lm 5,1-22 ; Dn 3,26-45 ; Is 38,10-[20] ; [Is 26,9-20] ; Hab 3,[2]-19 ; Prière de Manassé 115 ; Jon 2,3-11 et Jg 5,1-32. On remarquera l'absence du cantique des Trois Jeunes Gens. Des renseignements dont Verecundus émaille son commentaire, on peut extraire les données générales suivantes : les cantiques forment un seul livre avec le psautier, ils sont utilisés dans la liturgie, et il existe des listes divergentes. Le texte de tous ces cantiques est vieux latin, et de type africain.

\section{Le cantique des Trois Jeunes Gens (Dn 3,57-88[90])}

Les cantiques daniéliques apparaissent, en tradition directe, dans les séries de cantiques bibliques annexées aux psautiers ${ }^{15}$. Ils comptent parmi les pièces poétiques les plus utilisées dans l'Église chrétienne tant grecque et orientale ${ }^{16}$ que latine. Dans l'Église latine, les premiers regroupements ont dû avoir lieu vers la fin du $4^{\mathrm{e}}$ s., comme l'indiquent les témoignages patristiques. La plus ancienne série attestée en tradition directe, la série romaine, remonte à la seconde moitié du $5^{\mathrm{e}} \mathrm{s} .{ }^{17}$ Mais les cantiques avaient déjà une longue histoire derrière eux, comme l'atteste le caractère parfois archaïque de leur formulation latine.

Le choix des pièces et leur acolouthie ont largement varié au fur et à mesure que les différentes séries ont vu le jour, à savoir les séries romaine, irlandaise, milanaise, gallicane et mozarabe $^{18}$. Le cantique des Trois Jeunes Gens en Dn 3,57-88[90] est transmis sous forme vieille latine dans les séries romaine, gallicane ancienne, irlandaise et milanaise. Il est vulgate dans les cantiques annexés au psautier de type gallican (après la réforme carolingienne) et mozarabe, ainsi que dans les témoins de l'office monastique nocturne bénédictin.

Le répertoire de R. Gryson, en particulier dans le second tome, identifie près de soixantedix manuscrits qui transmettent sous forme vieille latine ${ }^{19}$ le cantique des Trois Jeunes Gens. Le texte du cantique variant très peu, surtout dans les très nombreux psautiers romains, il faudra opérer un choix pour ne pas gonfler inutilement l'apparat des témoins. Les manuscrits de l'époque carolingienne seront retenus. En revanche, à quelques exceptions près, les témoins postérieurs au $11^{\mathrm{e}} \mathrm{s}$. ne le seront pas. On veillera en tout cas à utiliser un nombre significatif de témoins des différentes séries ou de leurs recensions, en particulier pour les séries milanaises. Bon nombre de ces témoins ont été édités ou numérisés et sont disponibles sur Internet.

Les manuscrits de la série romaine (psautiers simples, doubles, triples ou quadruples) regroupent à eux seuls plus de la moitié des témoins répertoriés par R. Gryson. Seuls quatorze d'entre eux seront utilisés : 311 (Psautier de Salomon III, Saint-Gall, daté de 909, la série ro-

\footnotetext{
${ }^{14}$ Voir SCHNEIDER 1938, p. 17-18.

${ }^{15}$ Mais il importe de bien distinguer l'histoire des cantiques de celle des psautiers eux-mêmes : les conclusions tirées de l'histoire du psautier ne valent pas automatiquement pour les cantiques.

${ }^{16}$ Sur la présence des cantiques daniéliques dans les diverses Églises orientales, voir MEARNS 1914, p.25-49.

17 SCHNEIDER 1938, p. 60-61.

18 Voir les ouvrages de Mearns et Schneider cités plus haut.

${ }^{19}$ Certains manuscrits copient en parallèle les textes vieux latin et vulgate. On ne considère ici que les sections vieilles latines.
} 
maine est annexée au psautier romain) ${ }^{20}, 316$ (Psautier triple de Reichenau, deuxième quart du 9e s.), 344 (Mont-Cassin, 1099/1105), 354 (Bénévent ou Naples, fin du 11 e s.), 361 (origine italienne, fin du $11^{\mathrm{e}}$ s.), 368 (Nonantola, $11^{\mathrm{e}}$ s.), 372 (Psautier de Vespasien, sud de l'Angleterre, deuxième quart du $8^{\mathrm{e}}$ s.), 376 (Psautier de Cambridge, peut-être Saint-Augustin de Canterbury, vers 1000 ou peu après), 377 (Psautier Regius, peut-être Winchester, milieu du $10^{\mathrm{e}} \mathrm{s}$.), 383 (Psautier Bosworth, Canterbury, seconde moitié du 10 $\mathrm{s}$.), 393 (Psautier de Salaberga, Northumbrie, première moitié du $8^{\mathrm{e}} \mathrm{s}$.), 394 (provenant d'une fondation anglo-saxone sur le continent qui n'est toutefois pas Echternach, $8^{\mathrm{e}} \mathrm{s}$.), 395 (Fulda, premier tiers du $9^{\mathrm{e}} \mathrm{s}$.) et 399 (Bénévent, fin du $10^{\mathrm{e}} \mathrm{s}$.).

Parmi les témoins de la série gallicane ancienne, quatre témoins seront retenus : 7 (SaintGermain-des-Prés, vers 810), 250 (Psautier de Sedulius Scottus, milieu du 9e s.), 251 (Lectionnaire de Luxeuil, vers 700) et 341 (Psautier gréco-latin, Fulda, 9/10 $\mathrm{e}$ s.).

Trois témoins de la série irlandaise des cantiques seront utilisés : 254 (Antiphonaire de Bangor, entre 680 et 691), 255 (Irlande, début du $8^{\mathrm{e}}$ s.) et 257 (Irlande, fin du $11^{\mathrm{e}}$ ou début du $12^{\mathrm{e}} \mathrm{s}$.).

Les manuscrits de la série milanaise des cantiques se répartissent en trois recensions, celle des anciens Manualia, celle avec signes diacritiques et celle dite de Siméon. Des anciens $\mathrm{Ma}$ nualia seront repris 400 (Val Travaglia, 11 ${ }^{\mathrm{e}} \mathrm{s}$ ), 401 (Beroldus Novus, Milan, copié en 1268), 402 (Milan, daté de 1188) et 404 (Cernusco près de Milan, $11^{\mathrm{e}} \mathrm{s}$.). La recension avec signes diacritiques sera représentée par trois témoins : 405 (Milan, seconde moitié du $9^{\mathrm{e}} \mathrm{s}$.), 406 (Italie septentrionale, seconde moitié du $9^{e}$ s.) et 407 (Italie septentrionale, fin du $9^{\text {e }}$ s.). Deux témoins de la recension de Siméon seront pris en compte : 311 (Psautier de Salomon III, Saint-Gall, daté de 909, la série milanaise est annexée au psautier iuxta Hebraeos) et 408 (Milan, entre 858 et 899 ).

Restent trois témoins non rattachés spécifiquement aux séries énumérées plus haut : 300 (Psautier de Vérone, vers 600), 330 (Psautier de la Reine, Picardie, seconde moitié du $8^{\mathrm{e}} \mathrm{s}$.) et 460 (Psautier du Sinaï, date et origine inconnues).

En tradition indirecte, il y a bien l'une ou l'autre citation chez Augustin, Fulgence ou dans le De trinitate du pseudo-Vigile de Thaspe, mais aucune ne permet d'identifier un type de texte différent de celui des témoins manuscrits. Une première raison est à chercher dans la pauvreté littéraire du passage. Tous les versets sont construits sur le même schéma rudimentaire du point de vue syntaxique : benedicite $x$ dominum, hymnum dicite et superexaltate eum in saecula. Seul l'élément $x$ varie. La seconde en est le vocabulaire plutôt passe-partout de cet élément $x$. Des mots comme « cieux, anges, eaux, soleil, lune, étoiles, etc. », ne donnent pas lieu à variations nombreuses dans la traduction. À ne considérer que la formulation, aucune conclusion n'est donc possible. Mais peut-être faut-il prendre en compte un critère externe : la structure d'ensemble du cantique.

\footnotetext{
${ }^{20}$ Entre le $10^{\mathrm{e}} / 11^{\mathrm{e}} \mathrm{s}$. et le $13^{\mathrm{e}} \mathrm{s}$., bon nombre de psautiers ont été copiés directement ou indirectement sur le psautier de Salomon III, à savoir 310312313314315329409458461 462. " Trois de ces manuscrits sont des copies directes du psautier de Salomon, à savoir 314, 458 et 461 . Les manuscrits 315 et 462 ont été copiés respectivement sur 314 et 461 . Les manuscrits 312 et 310 sont des copies de 315 , mais le texte grec de 312 a été révisé, et 310 a peut-être été contaminé par 312. Sur 312 a été copié 313, qui a servi lui-même de modèle à 329 ; dans ce dernier manuscrit, le texte grec a été une nouvelle fois révisé, et le texte latin l'a été également par comparaison avec le grec. Enfin, 409 est une copie de 329 », GRYSON 1993-1997, p. 839. Il sera inutile de reprendre ces témoins puisque l'archétype est connu.
} 
L'ordre des créatures énumérées dans le cantique des Trois Jeunes Gens, du v. 57 au v. 88, a considérablement varié dans les traditions aujourd'hui conservées. Les variations apparaissent déjà dans les deux formes principales du texte biblique grec. Il n'est pas inutile de les présenter sous forme de tableau.

\begin{tabular}{|c|c|c|c|}
\hline & Théodotion (ms. A) & & LXX \\
\hline 57 & œuvres & 57 & œuvres \\
\hline 59 & cieux & 58 & anges \\
\hline 58 & anges & 59 & cieux \\
\hline 60 & eaux supérieures & 60 & eaux supérieures \\
\hline 61 & puissances & 61 & puissances \\
\hline 62 & soleir-lune & 62 & soleil-lune \\
\hline 63 & étoiles & 63 & étoiles \\
\hline 64 & pluie (ő $\mu \beta \rho \varsigma)$-rosée $(\delta \rho o ́ \sigma o \varsigma)$ & 64 & pluie (ő $\mu \beta \rho \circ \varsigma)$-rosée $(\delta \rho o ́ \sigma o \varsigma)$ \\
\hline 65 & vents $(\pi v \varepsilon v ́ \mu \alpha \tau \alpha)$ & 65 & vents $(\pi v \varepsilon v ́ \mu \alpha \tau \alpha)$ \\
\hline 66 & feu $(\pi \tilde{v} \rho)$-chaleur $(\kappa \alpha \tilde{v} \mu \alpha)$ & 66 & feu $(\pi \tilde{v} \rho)$-chaleur $(\kappa \alpha \tilde{\nu} \mu \alpha)$ \\
\hline 67 & 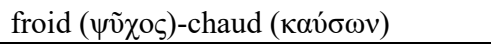 & 67 & froid ( $\dot{\rho} \tilde{i} \gamma o \zeta)$-hiver ( $\psi \tilde{\chi} \chi \circ \varsigma)$ \\
\hline 68 & 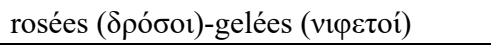 & 68 & 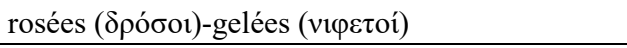 \\
\hline 71 & nuits-jours & 69 & gelées $(\pi \alpha ́ \gamma \eta)$-vent ( $\left.\psi v_{\chi} \eta\right)$ \\
\hline 72 & lumière-ténèbre & 70 & 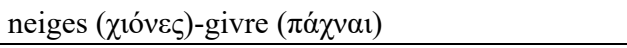 \\
\hline 69 & 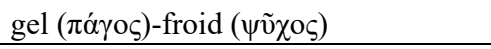 & 71 & nuits-jours \\
\hline 70 & givre ( $\pi \dot{\alpha} \chi v \alpha \imath)$-neiges $(\chi 1 o ́ v \varepsilon \varsigma)$ & 72 & ténèbre-lumière \\
\hline 73 & éclairs-nuages & 73 & éclairs-nuages \\
\hline 74 & terre & 74 & terre \\
\hline 75 & montagnes-collines & 75 & montagnes-collines \\
\hline 76 & produits de la terre & 76 & produits de la terre \\
\hline 78 & mers-fleuves & 77 & pluie $(o ̋ \beta \rho o t)$-source \\
\hline 77 & sources & 78 & mers-fleuves \\
\hline 79 & 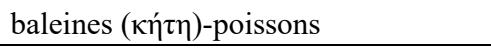 & 79 & 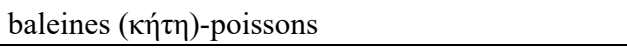 \\
\hline 80 & oiseaux & 80 & oiseaux \\
\hline 81 & bêtes sauvages ( $\theta \eta \rho i ́ \alpha)$-bétail ( $\kappa \tau \eta ́ v \eta)$ & 81 & quadrupèdes ( $\tau \varepsilon \tau \rho \alpha ́(\pi \circ \delta \alpha)$-bêtes sauvages $(\theta \eta \rho i ́ \alpha)$ \\
\hline 82 & hommes & 82 & hommes \\
\hline 83 & israhel & 83 & israhel \\
\hline 84 & prêtres & 84 & prêtres-serviteurs \\
\hline 85 & serviteurs & 85 & --- \\
\hline 86 & esprits-âmes des justes & 86 & esprits-âmes des justes \\
\hline 87 & saints-humbles de cœur & 87 & saints-humbles de cœur \\
\hline 88 & Ananias Azarias Misael & 88 & Ananias Azarias Misael \\
\hline
\end{tabular}

Ordre des créatures dans la tradition grecque

La LXX les énumère dans l'ordre arithmétique (ou plutôt, c'est l'ordre arithmétique qui a été choisi par les premiers auteurs de la répartition en versets, en commençant par la vulgate), mais elle réunit 84-85, en parlant de prêtres-serviteurs (sauf dans les témoins de la recension hexaplaire, 88 et Syh, où le texte est conformé à celui de Théodotion) (plus haut elle omet le 
v. 53). L'ordre des versets dans les témoins manuscrits de Théodotion ${ }^{21}$ et dans les versions qui en dépendent se caractérise par deux constantes : l'ordre 57.59.58 et l'inversion des versets $78.77^{22}$. Entre ces deux points fixes règne une grande variété, comme en témoigne le tableau qui suit.

\begin{tabular}{|l|l|l|}
\hline \multicolumn{1}{|c|}{ Ordre } & \multicolumn{1}{|c|}{ Témoins } & \multicolumn{1}{c|}{ Observation } \\
\hline $60-68.71-72.69-70.73-76$ & A 106 130 233 407 534 Bo Arab & \\
\hline $60-66.71-72.69-70.73-76$ & B-26 Q & omission de 67-68 \\
\hline $60-70.73 .72 .71 .74-76$ & 147 & \\
\hline $60-66.68 .71-72.69-70.73-76$ & $C$ & omission de 67 \\
\hline $60-65.71 .66 .68-69.72 .67 .70 .73-76$ & $46^{\prime}$ & \\
\hline $60-66.68 .71-72.67 .73-76$ & 239 & omission de 69-70 \\
\hline $60-66.68 .67 .71-72.70 .73-76$ & 410 & omission de 69 \\
\hline $60-68.71-72.70 .73-76$ & 541 Sah & omission de 69 \\
\hline $60-66.71 .68-69.72 .67 .70 .73-76$ & Eth & omission de 68-69 \\
\hline $60-67.70-76$ & Arm & transposition 61.62, omission de 68-69 \\
\hline $\begin{array}{l}61.60 .62-66.86 .71-72.67 .(+ \text { été- } \\
\text { hiver).70.73-76 }\end{array}$ & Syr & \\
\hline $60-65.67 .66 .68-70.73 .71-72.74 .76$ & Théodoret & \\
\hline
\end{tabular}

Ordre des versets de la section médiane dans la tradition grecque

Si on laisse de côté les témoins isolés, on se trouve en face de deux ordres principaux : celui de l'Alexandrinus (ms A, choisi par l'édition de Göttingen) et celui du Vaticanus (ms B). Les deux ne diffèrent que par l'omission des vv. 67-68 dans le second. Au vu de ces données, il n'y a rien d'étonnant à ce que la variété apparaisse également dans les témoins vieux latins.

Le plus ancien témoin de la série romaine des cantiques provient d'Angleterre ${ }^{23}$. Il s'agit du Psautier de Vespasien (VL 372) copié dans le deuxième quart du $8^{\mathrm{e}} \mathrm{s}$. Il contient les versets du cantique dans l'ordre suivant: 57.59.58.60-66.71-72.67.70.73-76.78.77.79-88.56. L'inversion des vv. 57.59 .58 correspond à l'ordre des versets dans la version grecque de Théodotion, de même que celle des vv. 78-77. En revanche, l'ordre 71-72.67 est particulier, ainsi que l'omission des vv. 68-69. Une donnée tout aussi remarquable est l'ajout du v. 56 à la fin du cantique. Cette disposition est sans correspondant dans le grec (voir tableau supra). En Angleterre, cet ordre du psautier romain est attesté également dans les témoins suivants classés chronologiquement : $394\left(8^{\mathrm{e}} \mathrm{s}\right.$ ), 377 (milieu du $10^{\mathrm{e}}$ s.) $383\left(2^{\mathrm{e}}\right.$ moitié du $10^{\mathrm{e}} \mathrm{s}$.) 376 (vers 1000 ) et 308 (vers 1140 , ce témoin ne sera pas retenu pour l'édition en raison de sa date tardive). Il est fort probable que cet ordre se retrouve en Germanie, comme pourrait l'indiquer le manuscrit 395 ( $1^{\text {er }}$ tiers du $9^{\text {e }} \mathrm{s}$.) ; le manuscrit est incomplet (vv.79-88.56), mais il se termine lui aussi sur le v. 56. Les témoins italiens du psautier romain avec cette disposition

${ }^{21}$ En laissant de côté ceux qui ont été revus sur le texte LXX, à savoir V-62 L'c c. Une tentative d'explication des divergences en Dn 3 dans les différentes formes des textes grecs (LXX, Théodotion, Papyrus 967) a été proposée par BOGAERT 1993.

22 Dans le bloc formé par les vv. 79-88, deux variations mineures sont attestées : l'omission du v. 85 dans le ms. 410, et l'inversion 84.83.82 dans le ms. 130. On peut les laisser ici de côté. Le ms 541 a l'ordre 70-7374.78.77.75.76.79.88 (il transpose 78.77 avant 75).

${ }^{23}$ Le lien souvent répété avec Canterbury n'a pas de fondement solide, voir GRYSON 1999 et 2004, ad loc. 
n'apparaissent qu'à partir du $11^{\mathrm{e}} \mathrm{s}$ : : $368\left(11^{\mathrm{e}} \mathrm{s}\right.$.) 344 (entre 1099 et 1105$) 347$ (entre 1099 et 1105, Mont-Cassin), 354 (fin du $11^{\mathrm{e}}$ s.), 343 ( $2^{\mathrm{e}}$ moitié du $12^{\mathrm{e}} \mathrm{s}$ ), 360 (vers 1150, Tivoli ; il omet le v. 82$), 370\left(12^{\mathrm{e}}\right.$ s., peut-être le Piémont) et $359\left(14^{\mathrm{e}}\right.$ s., Latium) (les quatre derniers témoins ne seront pas retenus). C'est la même disposition à Milan, dans les témoins de la recension avec signes diacritiques que sont $405\left(2^{\mathrm{e}}\right.$ moitié du $9^{\mathrm{e}} \mathrm{s}$.), $406\left(2^{\mathrm{e}}\right.$ moitié du $9^{\mathrm{e}} \mathrm{s}$.) et 407 (fin du $9^{\mathrm{e}} \mathrm{s}$.), et plus tard dans les anciens Manualia $400\left(11^{\mathrm{e}} \mathrm{s}\right.$ ), 401 (daté de 1268 ) et 402 (daté de 1188). Cette disposition est donc celle de la majorité des témoins manuscrits, psautiers comme livres liturgiques ${ }^{24}$.

En Italie, un témoin du $11^{\mathrm{e}}$ s., VL 361, a remanié cette disposition majoritaire et présente l'ordre suivant tout à fait exceptionnel : 57.59.58.63.60-62.64-65.71.66.72.67.74.70.73.7576.78-77.79-88.56. On y reconnaît les caractéristiques de la disposition majoritaire : vv. 5759.58, inversion des vv. 78-77 et présence du v. 56 en finale. Il est difficile de déceler une logique dans ces transformations.

En France, le Psautier de la Reine (VL $330,2^{\mathrm{e}}$ moitié du $8^{\mathrm{e}}$ s.) suit la disposition majoritaire, mais ne lit pas le v. 56 en fin de cantique.

Une modification supplémentaire est attestée en Irlande : le v. 56 est absent et on lit l'ordre vv. 83-82. Les témoins irlandais ont introduit aussi quelques modifications dans le vocabulaire : au v. 60 super caelos (au lieu de quae super caelos sunt); au v. 61 potentiae (au lieu de virtutes); au v. 66 calor (au lieu de aestus); au v. 81 iumenta (au lieu de universa pecora); au v. 79 beluae (au lieu de cete); au v. 83 israhelitae (et non israhel). Il lisent aussi fontes aquarum (au lieu de fontes) au v. 77, un ajout qui provient de Ps 17,$16 ; 41,2 ; 113,8^{25}$. Cette forme irlandaise est attestée par les manuscrits 254,255 et 257, ainsi que par le lectionnaire de Luxeuil (VL 251 ; le cantique ne commence qu'au v. 64 par suite de la perte d'un folio), qui témoigne vers 700 de l'arrivée des pièces liturgiques irlandaises sur le continent.

Parmi les psautiers gallicans anciens, le ms 7 (vers 810) a l'ordre majoritaire (57.59.58.6066.71-72.67.70.73-76.78.77.79-88), mais le v. 56 est remis à sa place et les vv. 52-56 forment la première partie du cantique des Trois Jeunes Gens (hymnus ananiae azariae misahel); le cantique est prolongé jusqu'au v. 90. Il fait également précéder le tout du cantique d'Azarias (vv. 26-45). En cela, il correspond exactement à la présentation des pièces du Psautier de Sedulius (VL 250, milieu du $9^{\mathrm{e}}$ s.). La prolongation du cantique jusqu'au v. 90 est attestée aussi par le témoin principal de la recension milanaise dite de Siméon, le ms. 408 (entre 858 et 899) ; et lui aussi fait précéder le cantique des Trois Jeunes Gens par le cantique d'Azarias, qu'il prolonge toutefois jusqu'au v. 51, auquel il adjoint immédiatement les vv. 52 à 56 . Dans ce témoin, chacune des trois pièces est intitulée ymnum trium puerorum. Mais à l'intérieur du Cantique des Trois Jeunes Gens, 408 dispose différemment les versets ; il a en effet l'ordre 57.59.58.60-67.64(bis).71-72.69.70.73-90. Il copie donc deux fois le v. 64 : une première fois

\footnotetext{
${ }^{24}$ Le psautier latin-grec VL 263 (12 $\mathrm{e}$ s., non retenu pour l'édition, mais numérisé sur Internet) contient les cantiques romains dont le texte vieux latin du cantique des Trois Jeunes Gens a été en partie aligné le texte grec. Le copiste rétablit l'ordre 57.58.59. Il abrège et regroupe les vv. 60-63 et le v. 80 (oiseaux) est omis. Le manuscrit $342\left(9^{\mathrm{e}} / 10^{\mathrm{e}}\right.$ s., non retenu, mais numérisé sur Internet) donne le texte d'une série de cantiques en grec translittéré accompagné d'une version latine interlinéaire. Pour le cantique des Trois Jeunes Gens, il a l'ordre unique suivant : 57.60-63.58-59. [en marge 64-65].66-67.70-88. Il est impossible de connaître l'emplacement exact des vv. 64-65 puisqu'ils ont été ajoutés dans la marge et qu'il n'y a aucune marque d'insertion. On observera le déplacement des vv. 60-63 entre les vv. 57 et 58, ainsi que l'omission habituelle du v. 69. À partir de v. 70 , l'ordre suit celui de la LXX (et donc de la vulgate).

25 Voir SCHNEIDER 1938, p. 97 (notes 34-35).
} 
après le v. 63 et une seconde fois après le v. 67 (qui, lui, est bien à sa place). Jusqu'à plus ample informé, il est le seul à copier le v. 69 et à ne pas inverser les vv. 77 et 78 .

Le psautier de Vérone (VL 300, vers 600) copie les versets dans l'ordre 57.59-66.7172.67-68.73-76.78.77.79-86.88. Il omet donc les vv. 58 et 87 (omissions tout à fait inhabituelles et peut-être accidentelles), ainsi que les vv. 69-70 (à vrai dire très semblables : gelfroid, givre-neige). De son côté, l'énigmatique psautier du Sinaï (VL 460) n'a pas le cantique à proprement parler, mais seulement les vv. vv 26-45 + 52-56 qui sont pourtant intitulés canticum trium puerorum.

Le critère externe, à savoir l'ordre des créatures énumérées, ne permet pas non plus d'identifier des types de texte. On se trouve en effet devant un système majoritaire: 57.59.58.60-66.71-72.67.70.73-76.78.77.79-88.56. Et il est quasiment impossible d'expliquer pourquoi certains témoins s'en écartent. Au total, les renseignements à tirer du cantique des Trois Jeunes Gens sont pauvres, ce qui est plutôt paradoxal pour un texte si abondamment transmis en tradition directe. La situation est radicalement différente pour le second cantique.

\section{Le cantique d'Azarias (Dn 3,26-45)}

Le cantique d'Azarias (3,26-45) a une attestation plus restreinte que le cantique des Trois Jeunes Gens. Il n'apparaît en effet que dans les témoins directs suivants : 7182250311 (et ses dérivés ${ }^{26}$ ) 330 408-409 et 460, et en tradition indirecte, chez Augustin et Verecundus qui le commentent, ainsi que chez Fulgence qui le cite en partie (vv. 26-31.39.40a.41.42a.45). Les témoins directs sont moins nombreux, mais les citations patristiques sont décisives pour mieux cerner l'histoire du texte.

Le manuscrit 7 (vers 810), témoin de l'ancienne liturgie gallicane, est incomplet; les cantiques suivants ont été conservés : Ex 15 (à partir de misisti iram au v. 7b), Hab 3, 1 Rg 2, Is 26, les cantiques d'Azarias et des Trois Jeunes Gens. Après le cantique des Trois Jeunes Gens, les mots explicit psalterium indiquent bien que les cantiques sont annexés au psautier. Le texte des cantiques est presque totalement identique à celui du Psautier copié par Sedulius vers le milieu du $9^{\mathrm{e}} \mathrm{s}$. (VL 250), ce qui suppose un modèle commun (précisions ci-après). Toutefois, en 250, la liste des cantiques est plus longue : Ex 15, Dt 32, Hab 3, 1 Rg 2, Is 26, cantique d'Azarias et des Trois Jeunes gens, Jon 2, Lc 1,46-55 (Magnificat) ; 1,68-79 (Zacharie) ; 2,29-32 (Nunc dimittis) ; Sedulius y a même ajouté une forme abrégée du cantique de la Vigne $^{27}$. Schneider affirme que l'archétype de $7+250$ est apparenté à la bible d'Augustin ${ }^{28}$. Une mise en parallèle du texte des principaux témoins permettra de vérifier cette dernière affirmation.

\begin{tabular}{|l|l|l|l|}
\hline & $7+250+330$ & 460 & \multicolumn{1}{c|}{ VER + FU } \\
\hline 26 & $\begin{array}{l}\text { benedictus es domine deus patrum } \\
\text { nostrorum et laudabile (-lis 250*) et } \\
\text { gloriosum nomen tuum in saecula }\end{array}$ & $\begin{array}{l}\text { benedictus es domine deus patrum } \\
\text { nostrorum et laudabilis et gloriosus } \\
\text { (om.) in saecula }\end{array}$ & $\begin{array}{l}\text { benedictus es domine deus patrum } \\
\text { nostrorum et laudabile (-lis } \mathrm{FU} \text { ) et } \\
\text { gloriosum (+ est VER) nomen tuum } \\
\text { in saecula }\end{array}$ \\
\hline
\end{tabular}

\footnotetext{
${ }^{26}$ Voir note 20.

${ }^{27}$ GRYSON 1993-1997, p. 818-819; GRYSON 2007, p. 349-350.

${ }^{28}$ SCHNEIDER 1938, p. 172-177.
} 


\begin{tabular}{|c|c|c|c|}
\hline & $7+250+330$ & 460 & $\mathrm{VER}+\mathrm{FU}$ \\
\hline 27 & $\begin{array}{l}\text { quoniam iustus es (est } 330 *) \text { in } \\
\text { omnibus quae fecisti nobis et omnia } \\
\text { opera tua et rectae viae tuae et } \\
\text { omnia iudicia tua }(\times \times \times \times \times \times \times \times \\
7250) \text { veritas }\end{array}$ & $\begin{array}{l}\text { quoniam iustus es in omnibus quae } \\
\text { fecisti nobis et omnia opera tua } \\
\text { veritas et rectae viae tuae et omnia } \\
\text { iudicia tua }\end{array}$ & $\begin{array}{l}\text { quoniam iustus es in omnibus quae } \\
\text { (quaecumque VER) fecisti nobis et } \\
\text { omnia opera tua ( }+ \text { vera FU) et } \\
\text { rectae viae tuae et omnia iudicia tua } \\
\text { veritas }(\times \times \times \times \times \text { VER) }\end{array}$ \\
\hline 28 & $\begin{array}{l}\text { et iudicia veritatis fecisti secundum } \\
\text { omnia quae adduxisti (induxisti 330) } \\
\text { nobis et super civitatem sanctam } \\
\text { patrum nostrorum hierusalem quo- } \\
\text { niam in veritate et iudicio fecisti } \\
\text { (induxisti 330) haec propter peccata } \\
\text { nostra (iniquitates nostras } 7250 \text { ) }\end{array}$ & $\begin{array}{l}\text { veritatis fecisti secundum omnia } \\
\text { quae adduxisti nobis et super } \\
\text { civitatem sanctam patrum } \\
\text { hierusalem quoniam in veritate et } \\
\text { iudicio fecisti haec omnia propter } \\
\text { peccatanostra }\end{array}$ & $\begin{array}{l}\text { et iudicia (-cium FU) veritatis fecisti } \\
\text { secundum omnia quae induxisti } \\
\text { nobis et super civitatem sanctam } \\
\text { patrum nostrorum Ierusalem quo- } \\
\text { niam in veritate et iudicio induxisti } \\
\text { haec omnia propter peccata nostra }\end{array}$ \\
\hline 29 & $\begin{array}{l}\text { quoniam peccavimus et inique egi- } \\
\text { mus discedere a te et perquam (mul- } \\
\text { tum 330) peccavimus in omnibus }\end{array}$ & $\begin{array}{l}\text { quoniam peccavimus et iniquitatem } \\
\text { fecimus in omnibus }\end{array}$ & $\begin{array}{l}\text { quoniam peccavimus et inique egi- } \\
\text { mus discedere (discedentes } \mathrm{FU} \text { ) a te } \\
\text { et multum peccavimus in omnibus }\end{array}$ \\
\hline 30 & $\begin{array}{l}\text { et mandatis tuis non audivimus } \\
\text { (obauivimus 330) neque conservavi- } \\
\text { mus neque fecimus sicut praecepisti } \\
\text { nobis ut bene nobis esset }\end{array}$ & $\begin{array}{l}\text { et mandatis tuis non obedivimus } \\
\text { neque conservavimus neque fecimus } \\
\text { sicut praecepisti nobis ut bene nobis } \\
\text { esset }\end{array}$ & $\begin{array}{l}\text { et mandatis tuis non oboedivimus } \\
\text { neque (nec FU) conservavimus } \\
\text { neque fecimus sicut praecepist } \\
\text { (praeceperas VER) nobis et bene } \\
\text { nobis esset }\end{array}$ \\
\hline 31 & $\begin{array}{l}\text { et omnia quaecumque nobis fecisti et } \\
\text { omnia }(\times \times \times \times \times 250) \text { quaecumque } \\
\text { (quae } 330) \text { adduxisti (induxisti 330) } \\
\text { nobis in vero iudicio fecisti }\end{array}$ & $\begin{array}{l}\text { et omnia quaecumque nobis fecisti et } \\
\text { omnia quae adduxisti in nobis in } \\
\text { vero iudicio fecisti }\end{array}$ & $\begin{array}{l}\text { et omnia quaecumque nobis fecisti et } \\
\text { omnia quae induxisti nobis ( quae } \\
\text { indux. nobis et omnia quaec. fecisti } \\
\text { nobis } \mathrm{FU}) \text { in vero iudicio fecisti }\end{array}$ \\
\hline 32 & $\begin{array}{l}\text { et tradidisti nos in manus (manibus } \\
250 *) \text { inimicorum iniquorum }(\times 250) \\
\text { inimicissimorum apostatarum }(\times 7) \\
\text { et regno iniquo et nequissimo prae } \\
\text { omni terra }\end{array}$ & $\begin{array}{l}\text { et tradidisti nos in manibus } \\
\text { inimicorum } \quad \text { inimicissimorum } \\
\text { apostat }<\text { ar }>\text { um et regno iniquo et } \\
\text { nequissimo prae omni tempore }\end{array}$ & $\begin{array}{l}\text { et tradidisti nos in manibus inimico- } \\
\text { rum iniquorum inimicissimorum } \\
\text { apostatarum et regno iniquo et } \\
\text { nequissimo prae omni terra }\end{array}$ \\
\hline 33 & $\begin{array}{l}\text { et nunc non est nobis aperire os } \\
\text { confusio et exprobratio facta est } \\
\text { servis tuis et colentibus te }\end{array}$ & $\begin{array}{l}\text { et nunc non est nobis aperire os } \\
\text { confusio et exprobratio facta est } \\
\text { servis tuis et colentibus te }\end{array}$ & $\begin{array}{l}\text { et nunc non est nobis aperire os } \\
\text { confusio et exprobratio facta est } \\
\text { servis tuis et colentibus te }\end{array}$ \\
\hline 34 & $\begin{array}{l}\text { et ne tradas nos in finem (fine } 330) \\
\text { propter nomen tuum et ne destruas } \\
\text { testamentum tuum }(\times \times \times \times \times 7 \\
330)\end{array}$ & $\begin{array}{l}\text { et ne tradas nos in fine propter } \\
\text { nomen tuum et ne disperdas } \\
\text { testamentum tuum }\end{array}$ & $\begin{array}{l}\text { et ne tradas nos in finem propter } \\
\text { nomen tuum (om.) }\end{array}$ \\
\hline 35 & $\begin{array}{l}\text { et ne avertas misericordiam tuam a } \\
\text { nobis propter abraham dilectum } \\
\text { tuum (dilectum a te 7) et propter } \\
\text { isaac servum tuum et israhel sanc- } \\
\text { tum tuum }\end{array}$ & $\begin{array}{l}\text { et ne auferas misericordiam tuam a } \\
\text { nobis propter abraham dilectum } \\
\text { tuum et propter isaac servum tuum et } \\
\text { israhel sanctum tuum }\end{array}$ & $\begin{array}{l}\text { et ne avertas misericordiam tuam a } \\
\text { nobis propter Abraham dilectum } \\
\text { tuum et Isaac servum tuum et Israel } \\
\text { sanctum tuum }\end{array}$ \\
\hline 36 & $\begin{array}{l}\text { quibus locutus es (est 330) adimplere } \\
\text { semen eorum quasi (sicut 330) stel- } \\
\text { las caeli et quasi (sicut 330) arenam } \\
\text { (arena } 330,+ \text { maris } 250 \text { ) quae ad } \\
\text { ora (oram } 250) \text { maris est }(\sim \text { est ad } \\
\text { ora maris } 330)\end{array}$ & $\begin{array}{l}\text { quibus locutus es adinplere semen } \\
\text { eorum quasi stellas caeli et velut } \\
\text { arena quae est ad ora maris }\end{array}$ & $\begin{array}{l}\text { quibus locuutus es adimplere semen } \\
\text { eorum sicut stellas coeli et sicut } \\
\text { arena quae est ad oram maris }\end{array}$ \\
\hline
\end{tabular}




\begin{tabular}{|c|c|c|c|}
\hline & $7+250+330$ & 460 & $\mathrm{VER}+\mathrm{FU}$ \\
\hline 37 & $\begin{array}{l}\text { quoniam domine minorati sumus } \\
\text { prae omnibus gentibus et sumus } \\
\text { humiles in omni terra (omnem ter- } \\
\text { ram 330) hodie propter peccata } \\
\text { nostra (iniquitates nostras } 7250 \text { ) }\end{array}$ & $\begin{array}{l}\text { quoniam domine minorati sumus } \\
\text { prae omnibus gentibus et sumus } \\
\text { humiles hodie propter peccata nostra }\end{array}$ & $\begin{array}{l}\text { quoniam minorati sumus domine } \\
\text { prae omnibus gentibus et sumus } \\
\text { humiles in omnem terram hodie } \\
\text { propter peccata nostra }\end{array}$ \\
\hline 38 & $\begin{array}{l}\text { et non est in tempore hoc ( hoc } \\
\text { tempore } 330) \text { princeps et propheta et } \\
\text { dux neque holocaustomata (-toma } 7) \\
\text { neque sacrificium neque oblatio (- } \\
\text { nes } 7, \times \times 330) \text { neque incensus (-um } \\
330) \text { nec (neque } 330) \text { locus ad sacri- } \\
\text { ficandum in conspectu tuo et inve- } \\
\text { nire misericordiam (-dia } 330 \text {, et in } \\
\text { veram misericordiam } 250)\end{array}$ & $\begin{array}{l}\text { et non est in tempore hoc princeps } \\
\text { neque propheta neque dux neque } \\
\text { holocaustamata neque sacrificium } \\
\text { neque oblationem neque incensum } \\
\text { nec locus ad sacrificandum in } \\
\text { conspectu tuo et invenire } \\
\text { misericordiam }\end{array}$ & $\begin{array}{l}\text { et non est in hoc tempore princeps et } \\
\text { propheta et dux neque holocausto- } \\
\text { mata neque sacrifi-cium neque obla- } \\
\text { tio neque incensum neque locus ad } \\
\text { sacrificandum in conspectu tuo et } \\
\text { invenire misericordiam }\end{array}$ \\
\hline 39 & $\begin{array}{l}\text { sed in anima contribulata et spiritu } \\
\text { humiliationis accipiamur (accipimus } \\
\text { nos 250) }\end{array}$ & $\begin{array}{l}\text { sed in anima } \text { co }<n>\text { tribulata spiritu } \\
\text { humilationis inveniamur }\end{array}$ & $\begin{array}{l}\text { sed in anima contribulata et spiritu } \\
\text { humiliationis accipiamur }\end{array}$ \\
\hline 40 & $\begin{array}{l}\text { quasi in holocausto (-tos } 7 \text {, -tis } 330) \\
\text { arietum et taurorum et multitudine (- } \\
\text { nem } 7 \text { ) agnorum pinguium sic fiat } \\
\text { sacrificium nostrum in conspectu tuo } \\
\text { hodie et perficere subsequentes te } \\
\text { quoniam non est confusio his ( } \times 7 \\
250) \text { qui confidunt }(* * * \text { confident } \\
\left.250^{*}, \times \text { confidentibus } 250^{c}\right) \text { confi- } \\
\text { dentibus in te }\end{array}$ & $\begin{array}{l}\text { tamquam in holocaustis arietum et } \\
\text { taurorum aut in multitudine agnorum } \\
\text { pinguium sic fiat sacrificium nostrum } \\
\text { in conspectu tuo hodie et perfice } \\
\text { subsequentes te quoniam non est } \\
\text { confusio his qui confidunt in te }\end{array}$ & $\begin{array}{l}\text { quasi in holocaustis arietum et tau- } \\
\text { rorum et multitudine agnorum pin- } \\
\text { guium sic fiat sacrificium nostrum in } \\
\text { conspectu tuo hodie et perfice } \\
\text { subsequentes te quoniam non est } \\
\text { confusio his qui confidunt in te }\end{array}$ \\
\hline 41 & $\begin{array}{l}\text { et nunc exsequimur in toto corde et } \\
\text { timemus te et inquirimus faciem } \\
\text { tuam }\end{array}$ & $\begin{array}{l}\text { nunc te sequimur in toto corde et } \\
\text { timemus te quaerimus faciem tuam }\end{array}$ & $\begin{array}{l}\text { et nunc exsequimur (sequimur } \mathrm{FU} \text { ) in } \\
\text { toto corde timemus te quaerimus } \\
\text { faciem tuam }\end{array}$ \\
\hline 42 & $\begin{array}{l}\text { ne confundas nos sed fac nobiscum } \\
\text { secundum modestiam (maiestatem } \\
250330 \text { ) tuam et secundum multitu- } \\
\text { dinem miserationum tuarum }\end{array}$ & $\begin{array}{l}\text { ne confundas nos sed fac nobiscum } \\
\text { secundum modestia tua et secundum } \\
\text { multitudinem misera-tionum tuarum }\end{array}$ & $\begin{array}{l}\text { ne confundas nos et fac nobiscum } \\
\text { secundum maiestatem tuam et } \\
\text { secundum multitudinem misera- } \\
\text { tionum tuarum }\end{array}$ \\
\hline 43 & $\begin{array}{l}\text { et libera nos secundum mirabilia tua } \\
\text { et da gloriam nomini tuo domine }\end{array}$ & $\begin{array}{l}\text { libera nos secundum multa mirabilia } \\
\text { tua et da gloriam nomini tuo domine }\end{array}$ & da gloriam nomini tuo domine \\
\hline 44 & $\begin{array}{l}\text { et confundantur omnes qui ostendunt } \\
\text { servis tuis mala et confundantur ab } \\
\text { omni potentia sua et virtus eorum } \\
\text { conteratur (virtutes eorum conteran- } \\
\text { tur } 250 \text { ) }\end{array}$ & $\begin{array}{l}\text { confundantur omnes qui ostendunt } \\
\text { servis tuis mala et confundantur ab } \\
\text { omni potentia sua et virtus eorum } \\
\text { conteratur }\end{array}$ & $\begin{array}{l}\text { confundantur omnes qui ostendunt } \\
\text { servis tuis mala et confundantur ab } \\
\text { omni potentia sua et virtus eorum } \\
\text { conteratur }\end{array}$ \\
\hline 45 & $\begin{array}{l}\text { et cognoscant quoniam tu es }(\times 250) \\
\text { dominus deus solus et gloriosus }(\times \times \\
330) \text { in omni orbe terrarum }\end{array}$ & $\begin{array}{l}\text { et cognoscant quoniam nomen tibi } \\
\text { dominus tu solus altissimus et } \\
\text { gloriosus in omni orbe terrarum }\end{array}$ & $\begin{array}{l}\text { et cognoscant quia (quoniam } \mathrm{FU} \text { ) tu } \\
\text { es dominus deus solus in omni orbe } \\
\text { terrarum }\end{array}$ \\
\hline
\end{tabular}

Textes vieux latins du cantique d'Azarias

Il est manifeste que les deux témoins $7+250$ remontent à un archétype commun, comme le montrent leur libellé quasiment identique, ainsi que l'omission accidentelle dans la seconde 
partie du v. 27 par un passage du même au même sur omnia opera tua Oomnia iudicia tua

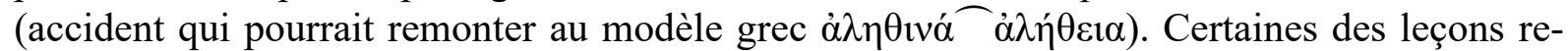
marquables de chacun des deux témoins s'expliquent par ce même type d'accident: ainsi l'omission de 250 au v. 31 (quaecumque quaecumque) et celle de 7 au v. 34 (tuum tuum). $\mathrm{Au}$ v. 32, les omissions de 7 et de 250 sont accidentelles (-rum -rum). On remarquera ce-

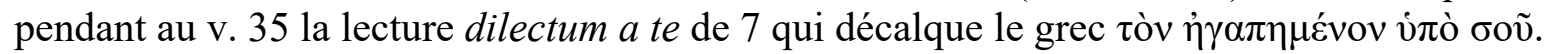

D'autres variations ont une moindre portée. Ainsi la variation laudabilis - laudabile (v. 26)

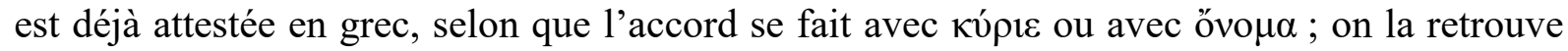
tout au long de la tradition latine. La substitution de manibus à manus (v. 32) est un phénomène fréquent dans la tradition latine (il suffit de consulter l'apparat de la vulgate pour s'en convaincre). Au v. 36, l'ajout de maris en 250 n'a aucun correspondant en grec ou dans la vulgate ; il en va de même pour le pluriel oblationes au verset suivant. Au v. 38, holocausto$m a$ en 7 est sans doute une erreur de copie, ce qu'est manifestement la leçon in veram misericordiam en 250. On considérera aussi comme des erreurs les mots accipimus nos au v. 39 en 250, ainsi que arietos et multitudinem en 7 au v. 40. Les variations de la fin du v. 40 en 7 et 250 (avant et après correction) s'expliquent par l'hésitation entre les deux leçons his qui con-

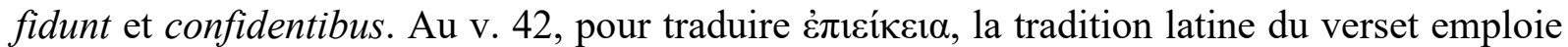
tranquillitas (VL 177 et CY), modestia (VL 7 et 460, accord remarquable) et mansuetudo (AU ep 111, la vulgate et les témoins qui en dépendent). Il est clair que la leçon maiestatem de 250, qui ne peut rendre le grec, est secondaire ; on la retrouve en 330, en 408 et chez Verecundus. Le pluriel (virtutes eorum conterantur) de 250 au v. 44 n'a pas de correspondant en grec ou dans la vulgate et est isolé dans la tradition vieille latine.

Le texte de 330 correspond dans les grandes lignes à celui de $7+260$, mais sans l'accident qui les caractérise au v. 27. On retrouve cependant au v. 34 le même accident qu'en 7 (tuum Tuum). Il n'a pas la leçon double sacrificium-oblatio de $7+250$ au v. 38, mais seulement sacrificium. Comme 250 il atteste la leçon maiestatem au v. 42.

Ces trois témoins remontent vraisemblablement à un même modèle latin du cantique dont les racines sont à chercher en Afrique du Nord comme l'indique la grande proximité avec les textes de Verecundus et Fulgence, telle qu'elle apparaît à la lecture du tableau ci-dessus. On notera parmi les variations suivantes que certaines semblent indiquer une parenté plus étroite entre 330 et VER + FU.

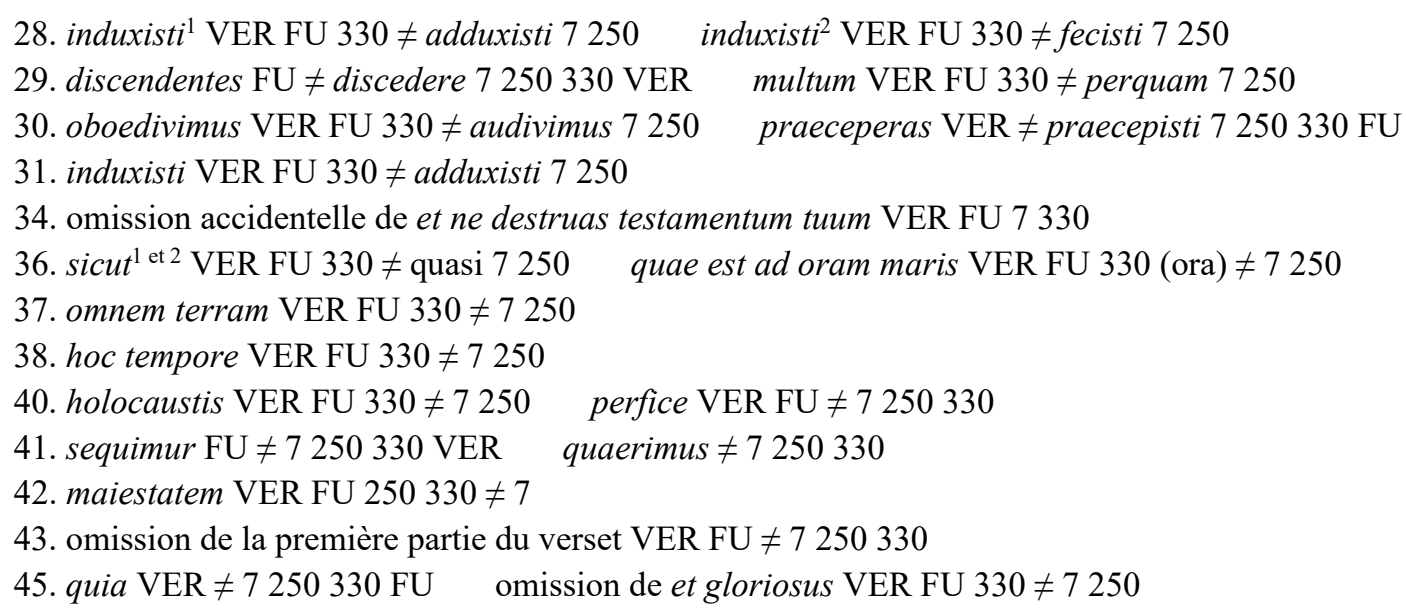

Qu'en est-il de 460 ? Sa proximité avec le texte africain est assez nette. Si on met à part les erreurs de lecture possibles et les omissions accidentelles par passage du même au même, il ne reste que quelques leçons propres. 
26. omission de nomen tuum

26-27. omission de veritas et iudicia tua (veritas veritatis)

29. iniquitatem fecimus $\neq 7250330 \mathrm{VER}$ FU omission de discedere a te et perquam peccavimus (fecimus (peccavimus)

32. omission de iniquorum (inimicorum iniquorum) prae omni tempore $\neq 7250330$ VER FU : par mauvaise résolution d'une abréviation?

34. disperdas $\neq 250$ (les autres témoins omettent la seconde partie du verset)

35. auferas $\neq 7250330$ VER FU : erreur de lecture?

36. velut $\neq 7250330$ VER FU

37. omission de in omni terra

38. oblationem $\neq 7250$ VER FU

40. holocaustis avec 330 perfice avec VER FU $\neq 7250330$

41. sequimur avec $\mathrm{FU} \neq 7250330 \mathrm{VER} \quad$ quaerimus avec VER $\mathrm{FU} \neq 7250330$

42. modestia $<m>$ tua $<m>$ avec $7 \neq 250330$ VER FU

43. ajout de multa devant mirabilia

45. ajout de nomen tibi altissimus et gloriosus $\neq 7250330 \mathrm{VER}$ FU : leçon double

Parmi les témoins manuscrits du cantique d'Azarias, VL 408, principal témoin de la recension milanaise dite de Siméon ${ }^{29}$, poursuit le cantique jusqu'au v. 51, rattachant ainsi les deux cantiques du chapitre 3 . Le texte du cantique diffère peu du texte africain. Voici les leçons notables (en comparaison du texte de 7250330 ).

26. laudabile et gloriosum nomen tuum in saecula] laudabilis et superexaltatus in saecula 408 : le cantique d'Azarias portant le titre hymnum trium puerorum, c'est le refrain du cantique des Trois Jeunes Gens qui est ici noté.

27. opera tua ] vera 408 avec FU et grec

28. adduxisti/induxisti] fecisti 408 omission de hierusalem

29. omission de perquam/multum

31. fecisti] induxisti 408 omission de in vero iudicio fecisti

32. regno iniquo et nequissimo] regi iniquo et pessimo 408

34. omission de la première partie du verset (tuum tuum) destruas] dispexeris 408

36. quasi...quasi] sicut...sicut 408 avec 330 VER FU

39. anima contribulata et spiritu humiliationis accipiamur] in anima spiritu humilitatis suscepit in nobis 408

40. fiat] faciat 408 : erreur de lecture? perficere] perfice 408 avec 460 VER FU

41. inquirimus] quaerimus avec $460 \mathrm{VER} F \mathrm{FU}$

42. modestiam] maiestatem avec 250330 VER FU

45. in omni orbe terrarum] in omnem orbem 408

Contrairement à ce qu'a affirmé Schneider, le modèle commun à tous ces témoins est toutefois différent du texte d'Augustin (AU ep 111,3 [646,2ss]). Les divergences apparaissent quasiment à chaque verset. On notera en particulier les leçons suivantes :

28. adduxisti...fecisti] intulisti...intulisti AU (cf v. 31)

29. inique egimus] legi non paruimus $\mathrm{AU}$

30. audivimus] obaudivimus $\mathrm{AU}$

31. fecisti...adduxisti/induxisti] intulisti...intulisti AU (cf v. 28)

32. apostatarum 250] transfugarum $\mathrm{AU}$ regno iniquo et nequissimo] regi iniusto et pessimo $\mathrm{AU}$ prae omni terrae] ultra universam terram $\mathrm{AU}$

33. aperire] ut possimus aperire $\mathrm{AU}$ exprobratio] opprobrium $\mathrm{AU}$ colentibus te] eis qui te colunt $\mathrm{AU}$

${ }^{29}$ Rappelons qu'elle est connue aussi par les manuscrits VL 311 (Psautier quadruple de Salomon III, SaintGall, daté de 909) et 409 (Cambridge, Corpus Christi College 468, 13e s., manuscrit non retenu pour l'édition). 
34. tradas] tradideris $\mathrm{AU} \quad$ finem] perpetuum $\mathrm{AU} \quad$ destruas] despexeris $\mathrm{AU}$

35. ne avertas] ne abstuleris $\mathrm{AU}$ dilectum a te] qui a te dilectus est $\mathrm{AU}$

36. adimplere] multiplicaturum te $\mathrm{AU} \quad$ quasi stellas] ut astra $\mathrm{AU}$ harenam quae ad ora maris est] harenam maris AU (cf 250)

37. minorati sumus] minimi facti sumus AU gentibus] nationibus

38. holocaustomata...sacrificium...oblatio...incensus] holocausta...oblatio...supplicationes AU

39. humiliationis] humilitatis AU

40. holocausto] holocaustomatibus AU

41. exsequimur] sequimur $\mathrm{AU} \quad$ inquirimus] quaerimus $\mathrm{AU}$

42. modestiam/maiestatem] mansuetudinem AU miserationum tuarum] misericordiae tuae AU

44. confundantur $\left.{ }^{1}\right]$ vereantur $\mathrm{AU}$

45. cognoscant] sciant in omni orbe terrarum] in universo orbe terrae AU

Le texte d'Augustin, qui cite manifestement à livre ouvert, n'est d'ailleurs pas exempt d'accident de copie, ainsi aux vv. 29-31 (quoniam peccavimus et legi non paruimus et mandatis tuis non obaudivimus ut bene nobis esset, et omnia quae intulisti nobis vero iudicio intulisti) dont la forme plus courte s'explique par des omissions par passage du même au même.

Le modèle africain commun à ces témoins ne correspond pas non plus à celui de Cyprien qui cite les vv. 37-42 en CY te 3,20. Le libellé de Cyprien varie considérablement comme le montrent les leçons suivantes.

38. et dux] neque potestas neque dux $\mathrm{CY} \quad$ holocaustomata] holocausta $\mathrm{CY} \quad$ sacrificium] hostia $\mathrm{CY}$ oblatio] thus $\mathrm{CY}$ ad sacrificandum] sacrificare $\mathrm{CY}$ in conspectu tuo] coram te $\mathrm{CY}$ misericordiam $]+$ a te $\mathrm{CY}$

39. omission de contribulata $\mathrm{CY}$ humiliationis] humilitatis $\mathrm{CY}$ accipiamur] acceptos nos habe $\mathrm{CY}$

40. quasi] ut $\mathrm{CY} \quad$ in holocaustis] holocaustos victimas $\mathrm{CY} \quad$ multitudinem] quasi multa milia $\mathrm{CY}$ pinguium] qui sunt pinguissimi $\mathrm{CY} \quad$ sacrificium nostrum] hostia nostra $\mathrm{CY}$ in conspectu tuo] coram te $\mathrm{CY} \quad$ perficere subsequentes te] consumetur potestas haec $\mathrm{CY} \quad$ non est confusio] non erubescent $\mathrm{CY}$

42. ne confundas nos] ne nos in obprobrium tradas $\mathrm{CY} \quad$ modestiam/maiestatem] tranquillitatem $\mathrm{CY}$ multitudinem] amplitudinem $\mathrm{CY}$ miserationum tuarum] misericordiae tuae $\mathrm{CY}$

Le texte européen vieux latin du cantique est au mieux représenté par le palimpseste de Würzburg (VL 177) ${ }^{30}$, qui n'est toutefois pas un témoin liturgique (pour ce qui nous en a été conservé, il donne en effet le texte suivi du livre de Daniel). Malgré cela, son témoignage peut être utilisé ici car il permet de préciser le schéma des types de texte pour le cantique d'Azarias. C'est en effet grâce à Lucifer de Cagliari qu'on peut prouver que le texte de 177 (avec 176 et 178) est européen. Certes, Lucifer de Cagliari ne cite aucun verset du cantique d'Azarias, mais ce n'est pas le cas pour d'autres passages du livre qu'il cite à longueur de page (voir en particulier l'Histoire de Suzanne et la vision des quatre bêtes au chapitre 7). Le texte de Lucifer (dont les œuvres sont datées des environs de 360) diffère à peine de celui de VL 176177 et 178, à tel point qu'on peut attribuer au texte de ces témoins le sigle D qui identifie habituellement le type de texte européen du milieu du $4^{\mathrm{e}}$ s. Voici, pour le cantique d'Azarias, les leçons caractéristiques de 177 (en comparaison du texte africain).

27. fecisti nobis] fecisti 177 opera tua] + vera 177

${ }^{30}$ À partir du v. 39, les fragments de Saint-Gall (VL 176) sont attestés, mais sous une forme très fragmentaire qui ne fait apparaître que deux différences par rapport à celui de 177 : sed] et 176 au v. 39 ; sequentes] subsequentes 176 au v. 40. 
27-28. omission de tua veritas et iudicia 177 : par passage du même au même

28. secundum omnia quae adduxisti/induxisti] per omnia quae intulisti nobis $177 \quad$ super civitatem sanctam] civitati sanctae 177 fecisti/induxisti] intulisti 177

29. inique egimus] legi non paruimus 177 avec $\mathrm{AU}$ discedere a te/discendentes a te] quod a te recessimus 177

30. mandatis tuis] peccavimus in omnibus mandatis tuis $177 \quad$ omission de neque fecimus $177 \quad$ sicut] ut 177

31. fecisti ... adduxisti/induxisti] intulisti ... fecisti 177 avec AU en partie

32. iniquorum] nostrorum 177 (la suite est illisible) regno iniquo et nequissimo] regi iniusto et pessimo 177 avec AU prae omni terra] praeter universam terram 177: mauvaise résolution d'une abréviation, voir AU (universam terram)

33. aperire] ut possimus aperire 177 avec $\mathrm{AU}$ confusio et exprobratio] verecundia et turpitudo 177 colentibus te] iis qui te colunt $177 \mathrm{cf} \mathrm{AU}$

34. tradas] tradideris 177 avec $\mathrm{AU}$ in finem] in perpetuum 177 avec $\mathrm{AU}$ omission de propter nomen tuum 177 destruas/disperdas] disperseris 177

35. ne avertas/auferas] ne abstuleris 177 avec $\mathrm{AU}$ dilectum tuum] qui a te dilectus est 177 avec $\mathrm{AU}$ omission de et israhel sanctum tuum 177 (tuum tuum)

36. adimplere] te multiplicaturum $177 \mathrm{cf} \mathrm{AU}$ ad ora(m) maris] circa ora maris 177

37. minorati sumus] minimi facti sumus 177 avec $\mathrm{AU} \quad$ prae omnibus gentibus] prae omnes nationes 177 (cf AU nationibus) omission de omnem devant terram et de nostra après peccata 177

38. oblatio] supplicationes 177 avec AU omission de neque incensum 177 avec AU misericordiam] + tuam 177

39. omission de contribulata $177 \quad$ humiliationis] humilitatis 177 avec $\mathrm{AU}$

40. quasi in holocaustis] ut in holocautomatibus 177 avec $\mathrm{AU}$ subsequentes avec 176] sequentes 177 non est] non erit 177 confusio] turpitudo $177 \quad$ qui confidunt in te] qui in te confidunt 177

42. modestiam/maiestatem] tranquillitatem 177 miserationum tuarum] misericordiae tuae 177 avec $\mathrm{AU}$

44. confundantur] vereantur 177 avec $\mathrm{AU}$

45. cognoscant ] sciant 177 avec $\mathrm{AU}$ in omni orbe terrarum] in universo orbe terrae 177 avec $\mathrm{AU}$

\section{Le cantique de la transcendance divine (Dn 3,52-55)}

$\mathrm{Ph}$. Bernard, dans son étude du cantique des Trois Jeunes Gens dans les répertoires liturgiques occidentaux ${ }^{31}$, distingue deux sections dans le cantique de Daniel : le cantique des créatures $(3,57-88)$ et une pièce qu'il appelle cantique de la transcendance divine $(3,52-55)$. Il a montré que, dans les répertoires liturgiques qu'il a étudiés ${ }^{32}$, l'immense majorité des manuscrits reprennent le cantique des créatures en entier; et ce sont, dans une écrasante proportion, des manuscrits français, aquitains et espagnols. Toutefois, à partir du début du $11^{\mathrm{e}} \mathrm{s}$., des manuscrits liturgiques allemands ont substitué à ce cantique le cantique de la transcendance divine. Cette pièce ne peut être que secondaire : non seulement elle est très écourtée, mais en plus les versets supprimés ont été remplacés par des passages empruntés ou inspirés d'autres livres bibliques. En effet, les mots benedictus es qui ambulas super pennas ventorum proviennent de Ps 103,3 ( $c f$. Ps 17,11), et la phrase benedictus es super sceptra divinitatis tuae est lointainement inspirée d'Ez 20,37 ou encore de Sag 10,13-14. ${ }^{33}$ Cette composition est cependant antérieure au $11^{\mathrm{e}}$ s., puisqu'elle est attestée par les psautiers milanais de la recension

${ }^{31}$ BERNARD 1993.

${ }^{32} \mathrm{Il}$ en énumère 62 aux p. 237-240.

${ }^{33}$ Ce passage du livre de la Sagesse réunit la mention du sceptre (au singulier, v. 14) et celle de la fosse (v. 13) (je remercie P. Bogaert qui a attiré mon attention sur cette dernière référence). Voir FERRETTI 1938, p. 213. 
avec signes diacritiques, VL 405406407 , qui datent tous trois de la seconde moitié du $9^{\mathrm{e}} \mathrm{s.}^{34}$ Elle se retrouvera plus tard dans les Manualia ambrosiens de l'office cathédral à partir du $11^{\mathrm{e}}$ s. (VL 400401402 ) où elle introduit l'office du matin depuis la période d'Ambroise ${ }^{35}$. Toutefois, au $9^{\mathrm{e}} \mathrm{s}$. encore, elle n'apparaît pas dans le principal témoin de la recension milanaise dite de Siméon, VL 408, qui donne le texte biblique authentique des versets 52-56. Schneider suppose que Siméon ne devait pas connaître cette pièce ${ }^{36}$. Mais ne pourrait-on pas envisager que l'auteur de cette rencension a rejeté cette pièce pour en revenir au texte biblique ? Cette hypothèse n'est pas invraisemblable quand on sait qu'il a procédé à deux autres changements importants : il a continué le cantique des créatures jusqu'au v. 90 et a fait précéder le tout du cantique d'Azarias (3,25-51). Comme Ph. Bernard le signale encore, le cantique des créatures a été également supplanté aux Quatre-Temps, par un Alleluia benedictus es (domine deus patrum nostrorum et laudabilis in saecula $)^{37}$ (Dn 3,52) et par un Omnipotentem semper adorent ${ }^{38}$, une composition poétique paraphrasant le texte du cantique due à Walafried Strabon (mort en 849). ${ }^{39}$

Voici le texte du cantique édité sur la base des témoins ambrosiens VL 400401402405 $406407^{40}$. Le lemme est celui de 405406407.

hymnus trium puerorum

benedictus es domine deus patrum nostrorum

et laudabilis et superexaltatus (gloriosus 400401402 ) in saecula

et benedictum nomen gloriae tuae quod est sanctum

et laudabile et superexaltatum (gloriosum 400401402 ) in saecula

benedictus es in templo sancto gloriae tuae

et laudabilis et superexaltatus (gloriosus 400401402 ) in saecula

benedictus es super sedem sanctam deitatis tuae

et laudabilis et superexaltatus (gloriosus 400401402 ) in saecula

benedictus es super thronum sanctum regni tui

et laudabilis et superexaltatus (gloriosus 400401402 ) in saecula

benedictus es super sceptra divinitatis tuae

et laudabilis et superexaltatus (gloriosus 400401 402) in saecula

benedictus es qui sedes super cherubim et seraphim

et laudabilis et superexaltatus (gloriosus 400401402 ) in saecula

benedictus es qui ambulas super pennas ventorum et super undas maris

${ }^{34}$ SCHNEIDER 1938, p. 70-74, a étudié l'utilisation du cantique daniélique aux Quatre-Temps et penchait pour une insertion milanaise tardive de cette pièce pour laquelle il supposait toutefois un modèle grec dénommée tractus.

${ }^{35}$ Le cantique des créatures est chanté le dimanche. Voir SCHNEIDER 1938, p. 9-10 et 100-101.

${ }^{36}$ SCHNEIDER 1938, p. 74.

37 À écouter sur : https://www.hommenouveau.fr/medias/files/Benedictus_es_Triors.mp3

${ }^{38}$ Voir le texte complet sur https://gregorien.info/chant/id/5971/13/en On peut écouter une composition contemporaine sur : https://www.youtube.com/watch?v=VTtZXJiTGkU

${ }^{39} \mathrm{Ph}$. Bernard s'exprime ainsi « Au total, à partir du XIème siècle, le 'Cantique des créatures' a été, dans trois mss. sur quatre, éliminé par trois rivaux: aux Quatre-Temps du quatrième mois, quand ils étaient rattachés à la semaine octave de la Pentecôte, par un Alleluia Benedictus es ; aux Quatre-Temps de septembre, par Omnipotentem; eux deux autres Quatre-temps, par le 'Cantique de la transcendance', principalement en Allemagne. C'est aux Quatre-Temps du premier mois qu'il a le mieux résisté, notamment dans les mss. français, aquitains et espagnols » (p. 240-241).

${ }^{40}$ Le refrain et laudabilis et superexaltatus in secula est parfois noté sous une forme abrégée qui n'est pas signalée ici. 
et laudabilis et superexaltatus (gloriosus 400401402 ) in saecula

(var. + benedicite omnia opera domini domino)

benedicant te omnes angeli et sanctu tui

et laudabilis et superexaltatus (gloriosus 400401402 ) in saecula

(+ benedicant te omnes angeli et sancti tui

et laudabilis et superexaltatus in saecula $407 *$ )

benedicant te caeli terra mare et omnia quae in eis sunt

et laudabilis et superexaltatus (gloriosus 400401402 ) in saecula

En conclusion, des trois cantiques transmis par la tradition latine en rapport avec le supplément du chapitre 3 de Daniel, seul celui d'Azarias $(3,26-45)$ contribue de manière significative à la mise en lumière des types de texte. En effet, le cantique des Trois Jeunes Gens (3,57-88[90]), avec sa syntaxe rudimentaire et son vocabulaire passe-partout, varie très peu en tradition tant directe qu'indirecte (les rares citations patristiques sont inexploitables). De son côté, le cantique de la transcendance divine $(3,52-56)$ n'entre pas véritablement dans le cadre d'une édition des vieilles versions latines de Daniel, étant donné que cette libre composition intègre des éléments empruntés à d'autres passages de la Bible. La variété du vocabulaire des témoins directs et indirects, jointe au fait que plusieurs écrivains ecclésiastiques citent à longue page ou commentent le texte du cantique d'Azarias, permettront d'écrire pour celui-ci au moins trois des grandes lignes majeures habituelles de l'édition de Beuron (sans compter la ligne vulgate $\mathbf{V}$ qui clôt le schéma de l'édition). Pour les vv. 37-42, le texte de Cyprien sera noté en ligne majeure $\mathbf{K}$, représentant le type de texte africain ancien (milieu du $3^{\mathrm{e}} \mathrm{s}$.). On pourra attribuer au texte transmis par 7250330 Verecundus et Fulgence le sigle $\mathbf{C}$, réservé aux auteurs africains tardifs. Le texte des témoins 176 et 177 (dont le libellé est proche de celui de Lucifer de Cagliari dans d'autres passages du livre de Daniel) sera identifié par le sigle D correspondant au texte européen des environs de 350. Le texte d'Augustin diffère considérablement du texte africain tardif; il n'est cependant pas identique au texte européen du milieu du $4^{\mathrm{e}} \mathrm{s}$. bien qu'il ait avec lui des leçons communes. Les leçons particulières de son texte apparaîtront en variante sous la ligne majeure $\mathbf{D}$ avec le sigle $\mathbf{A}$. 


\section{MANUSCRITS VIEUX LATINS CITÉS}

\begin{tabular}{|c|c|}
\hline VL & Bibliothèque, origine, date (avec éventuellement nom traditionnel du manuscrit) \\
\hline 7 & $\begin{array}{l}\text { Paris, Bibliothèque Nationale lat. } 11553 \text { (olim Sangermanensis 15); Saint-Germain-des Prés, vers } \\
810\end{array}$ \\
\hline 175 & $\begin{array}{l}\text { Darmstadt, Hessische Landes- und Hochschulbibliothek } 895+3140 \text { (précédemment 896) + Do- } \\
\text { naueschingen, Hofbibliothek B. I. 3, précédemment } 191+\text { Fulda, Landesbibliothek Aa 1a + Sankt } \\
\text { Paul im Lavanttal, Stiftsbibliothek sine numero + Stuttgart, Württembergische Landesbibliothek } \\
\text { fragm. } 100 \text {; Italie du Nord, } 5^{\mathrm{e}} \text { s. }\end{array}$ \\
\hline 176 & $\begin{array}{l}\text { Sankt Gallen, Stiftsbibliothek } 1397 \text { IV + 1398a + 1398b + Zürich, Zentralbibliothek C } 184 \text { (389); } \\
\text { Italie du Nord, premières décennies du } 9^{\mathrm{e}} \mathrm{s} \text {. }\end{array}$ \\
\hline 177 & Würzburg, Universitätsbibliothek M. p th. f. 64a ; Luxeuil, vers 700 \\
\hline 182 & Verona, Biblioteca Capitolare II (2) + XXXVII (35) ; origine incertaine, début du $7^{\mathrm{e}} \mathrm{s}$. \\
\hline 250 & Paris, Bibliothèque de l'Arsenal 8407 (Psautier de Sedulius), milieu du $9^{\mathrm{e}} \mathrm{s}$. \\
\hline 251 & Paris, Bibliothèque Nationale lat. 9427 (Lectionnaire de Luxeuil) ; vers 700 \\
\hline 254 & Milano, Biblioteca Ambrosiana C. 5 inf. (Antiphonaire de Bangor) ; Bangor, entre 680 et 691 \\
\hline 255 & Torino, Biblioteca Nazionale Universitaria F. IV. 1 fasc. 9 ; Irlande, début du $9^{\mathrm{e}} \mathrm{s}$. \\
\hline 257 & Killiney, Franciscan Library sine numero; fin $11^{\mathrm{e}} \mathrm{s}$. \\
\hline 263 & Città del Vaticano, Biblioteca Apostolica Vaticana Vatic. lat. $81 ; 12^{\mathrm{e}} \mathrm{s}$. \\
\hline 300 & Verona, Biblioteca Capitolare I (1) (Psautier de Vérone); Italie du Nord, vers 600 \\
\hline 308 & $\begin{array}{l}\text { Paris, Bibliothèque Nationale de France lat. } 8824 \text { (Psautier du Duc de Berry); Canterbury (?), vers } \\
1140\end{array}$ \\
\hline 310 & Monte Cassino, Archivio della Badia $467 \mathrm{BB} ; 12^{\mathrm{e}} \mathrm{s}$. \\
\hline 311 & Bamberg, Staatliche Bibliothek Bibl. 44 (A. I. 14) (Psautier de Salomon III) ; Saint-Gall, en 909 \\
\hline 312 & Paris, Bibliothèque Nationale de France nouv. acq. lat. 2195 ; Nord de la France, en 1105 \\
\hline 313 & Valenciennes, Bibliothèque Municipale 14 (7); Nord de la France, entre 1145 et 1153 \\
\hline 314 & Köln, Erzbischöfliche Diözesan- und Dombibliothek 8 ; Bamberg, deuxième quart du $11^{\mathrm{e}} \mathrm{s}$. \\
\hline 315 & Essen, Archiv des Münsters sine numero; fin du $11^{\mathrm{e}} \mathrm{s}$. \\
\hline 316 & Karlsruhe, Badische Landesbibliothek Aug. XXXVIII ; Reichenau, deuxième quart du $9^{\mathrm{e}} \mathrm{s}$. \\
\hline 329 & Paris, Bibliothèque Nationale de France lat. 15198 ; vers 1200 \\
\hline 330 & $\begin{array}{l}\text { Città del Vaticano, Biblioteca Apostolica Vaticana Regin. lat. } 11 \text { (Psautier de la Reine) ; Picardie, } \\
\text { seconde moitié du } 8^{\mathrm{e}} \mathrm{s} \text {. }\end{array}$ \\
\hline 341 & Cues an der Mosel, Bibliothek des St. Nikolaus-Hospitals 10 ; Fulda, 9 e $/ 10^{\mathrm{e}} \mathrm{s}$. \\
\hline 342 & Città del Vaticano, Biblioteca Apostolica Vaticana Regin. lat. $1595 ; 9^{\mathrm{e}} / 10^{\mathrm{e}} \mathrm{s}$. \\
\hline 343 & $\begin{array}{l}\text { Città del Vaticano, Biblioteca Apostolica Vaticana Vatic. lat. } 4928 \text {; Bénévent, seconde moitié du } \\
12^{\mathrm{e}} \mathrm{s} \text {. }\end{array}$ \\
\hline 344 & $\begin{array}{l}\text { Città del Vaticano, Biblioteca Apostolica Vaticana Urbin. lat. } 585 \text {; Mont-Cassin, entre } 1099 \text { et } \\
1105\end{array}$ \\
\hline 347 & Paris, Bibliothèque Mazarine 364 (Bréviaire de l'abbé Odérise) ; Mont-Cassin, entre 1099 et 1105 \\
\hline 354 & Città del Vaticano, Biblioteca Apostolica Vaticana Regin. lat. 13 ; Bénévent, fin du $11^{\mathrm{e}} \mathrm{s}$. \\
\hline 359 & $\begin{array}{l}\text { Città del Vaticano, Biblioteca Apostolica Vaticana Vatic. lat. } 10000 \text {; Otricoli (Nord de Rome), } \\
14^{\mathrm{e}} \mathrm{s} \text {. }\end{array}$ \\
\hline 360 & Città del Vaticano, Biblioteca Apostolica Vaticana Archivio di S. Pietro D. 156 ; Tivoli, vers 1150 \\
\hline 361 & Città del Vaticano, Biblioteca Apostolica Vaticana Archivio di S. Pietro D. 150 ; fin $11^{\mathrm{e}} \mathrm{s}$. \\
\hline 368 & Città del Vaticano, Biblioteca Apostolica Vaticana Vatic. lat. 84 ; Nonantola, $11^{\mathrm{e}} \mathrm{s}$. \\
\hline 370 & Città del Vaticano, Biblioteca Apostolica Vaticana Palat. lat. $30 ;$ Piémont (?), $12^{\mathrm{e}} \mathrm{s}$. \\
\hline 372 & $\begin{array}{l}\text { London, British Library Cotton Vespasian A. } 1 \text { (Psautier de Vespasien) ; Sud de l'Angleterre (vrai- } \\
\text { semblablement pas Canterbury), deuxième quart du } 8^{\mathrm{e}} \mathrm{s} \text {. }\end{array}$ \\
\hline
\end{tabular}




\begin{tabular}{|c|c|}
\hline VL & Bibliothèque, origine, date (avec éventuellement nom traditionnel du manuscrit) \\
\hline 376 & Cambridge, University Library Ff. I. 23 ; Canterbury (?), vers 1000 \\
\hline 377 & London, British Library Royal 2. B. 5 (Regius Psalter); Winchester (?), milieu du $10^{\mathrm{e}} \mathrm{s}$. \\
\hline 383 & London, British Library Add. 37517 (Bosworth Psalter) ; Canterbury, seconde moitié du $10^{\mathrm{e}} \mathrm{s}$. \\
\hline 393 & $\begin{array}{l}\text { Berlin, Staatsbibliothek Preussischer Kulturbesitz Hamilton } 553 \text { (Psautier de Salaberga) ; Northum- } \\
\text { brie, première moitié du } 8^{\mathrm{e}} \mathrm{s} \text {. }\end{array}$ \\
\hline 394 & Stuttgart, Württembergische Landesbibliothek Bibl. fol. 12a.b.c ; Echternach (?), $8^{\mathrm{e}} \mathrm{s}$. \\
\hline 395 & $\begin{array}{l}\text { Frankfurt am Main, Stad- und Universitätsbibliothek Ms. Bart. } 32 \text { (Ausst. 15) ; Fulda, premier tiers } \\
\text { du } 9^{\mathrm{e}} \mathrm{s} \text {. }\end{array}$ \\
\hline 399 & Napoli, Biblioteca Nazionale VI. F. 2 ; Bénévent, $10^{\mathrm{e}} \mathrm{s}$. \\
\hline 400 & Milano, Biblioteca Capitolare $4^{\circ} 6$ (D. 2.30 ; précédemment 2102); Val Travaglia, $11^{\mathrm{e}} \mathrm{s}$. \\
\hline 401 & Milano, Biblioteca Capitolare Beroldus Novus ; copié en 1268 \\
\hline 402 & Milano, Biblioteca Ambrosiana A. 189 inf. ; Milan, daté de 1188 \\
\hline 404 & Milano, Biblioteca Ambrosiana I. 55 sup. ; Cernusco (près de Milan), $11^{\mathrm{e}} \mathrm{s}$. \\
\hline 405 & München, Bayerische Staatsbibliothek Clm 343 ; Milan, seconde moitié du $9^{\mathrm{e}} \mathrm{s}$. \\
\hline 406 & $\begin{array}{l}\text { Città del Vaticano, Biblioteca Apostolica Vaticana Vatic. lat. } 83 \text {; Italie septentrionale, seconde } \\
\text { moitié du } 9^{\mathrm{e}} \mathrm{s} \text {. }\end{array}$ \\
\hline 407 & Città del Vaticano, Biblioteca Apostolica Vaticana Vatic. lat. 82 ; Nord de l'Italie, fin du 9 e s. \\
\hline 408 & Berlin, Staatsbibliothek Preussischer Kulturbesitz Hamilton 552 ; Milan, entre 858 et 899 \\
\hline 409 & Cambridge, Corpus Christi College 468 (N. 15); $13^{\mathrm{e}} \mathrm{s}$. \\
\hline 458 & $\begin{array}{l}\text { Bamberg, Staatsarchiv A 246, Nr. 20. I-III + Coburg, Staatsarchiv Fragm. } 2 \text { + Freiburg, Univer- } \\
\text { sitätsbibliothek } 629 \text {; Banz, vers } 1100\end{array}$ \\
\hline 460 & Sinaï, Monastère Sainte-Catherine slavon 5 (Psautier du Sinaï); origine et date inconnues \\
\hline 461 & Admont, Stiftsbibliothek 42 ; Admont, $12^{\mathrm{e}} \mathrm{s}$. \\
\hline 462 & Graz, Universitätbibliothek 86 ; Seckau, première moitié du $12^{\mathrm{e}} \mathrm{s}$. \\
\hline
\end{tabular}




\section{BIBLIOGRAPHIE}

BeRnARD Ph., 1993 : «Le Cantique des Trois Enfants (Dan. III, 52-90) et les répertoires liturgiques occidentaux dans l'Antiquité tardive et le haut Moyen Âge ", dans Musica e storia 1 , p. 231-272.

Bogaert P., 1993 : "Daniel 3 LXX et son supplément grec », dans A.S. van der Woude (ed.), The Book of Daniel in the Light of New Findings (Bibliotheca Ephemeridum Theologicarum Lovaniensium 106), Leuven, p. 13-37.

— 2004 : «La prière de Daniel (9,4-19) selon le Psautier latin du Sinaï. Un remaniement liturgique », dans Revue Bénédictine 114, p. 349-362.

- 2010 : «Les cantiques bibliques dans 1'homilétique africaine. Quelques perspectives de recherche », dans G. Partoens, A. Dupont, M. Lamberigts (eds), Ministerium Sermonis. Proceedings of the International Colloquium on St. Augustine's Sermones ad Populum. Turnhout-Leuven, May 29-31, 2008 (Instrumenta Patristica et Mediaevalia 53), Turnhout, p. 121-142.

DolD A., 1947 : «Die vom Missale Romanum abweichenden Lesetexte für die Messfeiern nach den Notierungen des aus Monte Cassino stammenden Codex Vat. Lat. 6082 », dans Vir Dei Benedictus. Eine Festgabe zum 1400. Todestag des heiligen Benedikt, Münster, p. 293-332.

FERRETTI P., 1938 : Esthétique grégorienne ou Traité des formes musicales du chant grégorien, Solesmes.

GRYSON R., 1993-1997 : Esaias (Vetus Latina. Die Reste der altlateinischen Bibel, 12), Freiburg.

- 1999 et 2004 : Altlateinischen Handschriften. Manuscrits latins. Répertoire descriptif. Première partie: Mss 1-275. Deuxième partie: Mss 300-485 (Manuscrits du psautier) (Vetus Latina. Die Reste der altlateinischen Bibel, 1/2B), 2 vol., Freiburg.

- 2007 : Répertoire général des auteurs ecclésiastiques latins de l'Antiquité et du Haut Moyen Âge (Vetus Latina. Die Reste der altlateinischen Bibel, 1), 2 vol., Freiburg.

HARL H., 2014 : Voix de louange. Les cantiques bibliques dans la liturgie chrétienne, avec la collaboration de Br. Meynadier et A. Pietrobelli (coll. Anagôgê), Paris.

MEARns J., 1914 : The Canticles of the Christian Church Eastern and Western in Early and Medieval Times, Cambridge.

SCHMITT A., 1996 : « Stammt der sogenannte « $\theta$ " -Text bei Daniel wirklich von Theodotion? », dans Nachrichten der Akademie der Wissenschaften in Göttingen. I. Philologischhistorische Klasse, 1966, p. 279-392 (repris dans Mitteilungen des SeptuagintaUnternehmens [MSU] 9), Göttingen, p. 1-114.

SCHNEIDER H., 1938 : Die altlateinischen biblischen Cantica (Texte und Arbeiten, 29-30), Beuron.

— 1949a : «Die biblischen Oden im christlichen Altertum », dans Biblica 30, p. 28-65.

— 1949b : «Die biblischen Oden seit dem sechsten Jahrhundert », Biblica 30, p. 239-272.

— 1949c : « Die biblischen Oden in Jerusalem und Konstantinopel », Biblica 30, p. 433-500.

Souter A., 1926 : Pelagius's Expositions of Thirteen Epistles of St. Paul, II : Texts and Apparatus Criticus (Texts and Studies 9/2), Cambridge. 
Ziegler J., MunNich O., Fraenkel D., 1999 : Susanna - Daniel - Bel et Draco edidit J. ZIEGLER. Editio secunda versionis iuxta LXX interpretes textum plane novum constituit $\mathrm{O}$. MunNICH. Versionis iuxta "Theodotionem » fragmenta adiecit D. FRAENKEL (Septuaginta. Vetus Testamentum Graecum Auctoritate Academiae Scientiarum Gottingensis editum XVI,2), Göttingen. 


\title{
RÉSUMÉ
}

Le livre de Daniel a transmis deux grands cantiques bibliques : le cantique d'Azarias (Dn 3,26-45) et le cantique des Trois Jeunes Gens (Dn 3,57-88[90]). Un troisième, plus petit, vient se glisser entre les deux : le cantique de la transcendance divine (Dn 3,52-56). Le texte vieux latin des deux grands cantiques est ici étudié dans le but d'y repérer les types de texte. Grâce aux témoins directs et indirects, en particulier pour le cantique d'Azarias, il est possible d'identifier un texte africain ancien $\mathbf{K}$ (transmis par Cyprien), un texte africain tardif $\mathbf{C}$ (7 250 330 Verecundus et Fulgence), un texte européen D (176 177 dont le libellé est proche de celui de Lucifer de Cagliari), un texte A (leçons particulières d'Augustin). L'article montre que les créatures énumérées dans le cantique des Trois Jeunes Gens se présentent dans un ordre très variable dans la tradition vieille latine, sans qu'il soit possible de déceler une quelconque logique.

\begin{abstract}
The book of Daniel has transmitted two long biblical canticles: the Prayer of Azariah (Dan 3,26-45) and the Song of the Three Children (Dan 3,57-88[90]); a third shorter canticle has been slipped in between the two others: the song of divine transcendence (Dan 3,52-56). The Old Latin text of the two long canticles is examined here in order to determine the text types. Thanks to direct and indirect witnesses, in particular for the Prayer of Azariah, it is possible to identify an old African text type K (transmitted by Cyprian), a later African text type $\mathbf{C}$ ( 7 250330 Verecundus and Fulgentius), a European text type D (176 177, the wording of which is close to that of Lucifer of Cagliari), a text type A (readings proper to Augustin). The article shows that the creatures enumerated in the Song of the Three Children are given in very variable orders in the Old Latin tradition without it being possible to detect any kind of logic.
\end{abstract}

